Tecno Lógicas

ISSN 0123-7799

Vol. 20, No. 38 , pp. $71-82$

Enero-junio de 2017

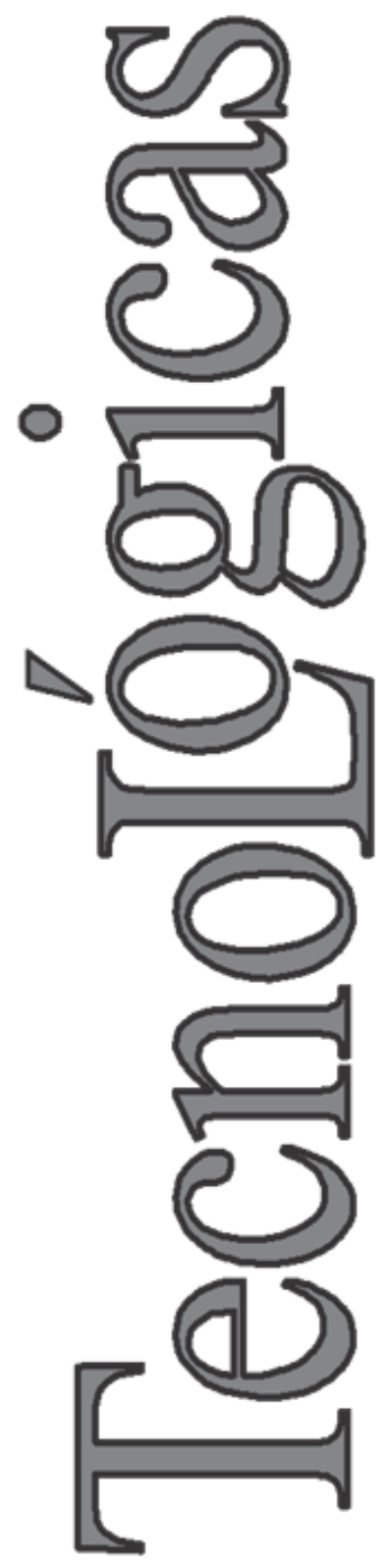

(C) Copyright 2015 por autores y Tecno Lógicas Este trabajo está licenciado bajo una Licencia Internacional Creative Commons Atribución (CC BY)

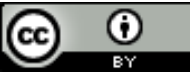

\section{Respuesta de la Cúrcuma (Curcuma longa L.) a la aplicación de un bio- fertilizante tratado con un campo magnético}

\section{Response of Turmeric (Curcuma longa L.) to the application of a bio-fertilizer treated with a magnetic field}

\author{
Orlando Zúñiga-Escobar ${ }^{1}$, \\ Cristian O. Jiménez-Alfaro ${ }^{2}$, Jhony A. Benavides- \\ Bolaños ${ }^{3}$ y Celina Torres-González ${ }^{4}$
}

Recibido: 12 de septiembre de 2015, Aceptado: 19 de septiembre de 2016

Cómo citar / How to cite

Orlando Zúñiga-Escobar, Cristian O. Jiménez-Alfaro, Jhony A. Benavides-Bolaños y Celina Torres-González, "Respuesta de la Cúrcuma (Curcuma longa L.) a la aplicación de un bio-fertilizante tratado con un campo magnético", Tecno Lógicas, vol. 38, no. 20, pp. $71-82$ enero - junio, 2017.

1 Doctor en Tecnología Agroambiental, Grupo de Investigación en Ciencias Ambientales y de la Tierra ILAMA, Departamento de Física, Facultad de Ciencias Naturales y Exactas, Universidad del Valle, Cali-Colombia, orlando.zuniga@correounivalle.edu.co

2 Ingeniero Agrícola, Grupo de Investigación en Ciencias Ambientales y de la Tierra ILAMA, Departamento de Física, Facultad de Ciencias Naturales y Exactas, Universidad del Valle, Cali-Colombia, cristian.o.jimenez@correounivalle.edu.co

3 Ingeniero Agrícola, Grupo de Investigación en Ciencias Ambientales y de la Tierra ILAMA, Departamento de Física, Facultad de Ciencias Naturales y Exactas, Universidad del Valle, Cali-Colombia, jhony.benavides@correounivalle.edu.co

4 MSC. Ingeniera Agrónoma, Grupo de Investigación en Biología de Plantas y Microorganismos, Departamento de Biología Facultad de Ciencias Naturales y Exactas, Universidad del Valle, Cali-Colombia, celina.torres@correounivalle.edu.co 


\section{Resumen}

Se evaluó la respuesta del desarrollo y la producción de la cúrcuma (Curcuma longa L.) a la aplicación de una mezcla en solución del bio-fertilizante Multibiol® y agua potable, tratada con una inducción de flujo magnético de $25 \mathrm{mT}$ AC con una frecuencia de $60 \mathrm{~Hz}$. Se utilizó un circuito de recirculación conectado a un solenoide. La solución se aplicó a una concentración de 1:12 durante tres meses a plántulas bajo condiciones de invernadero, en dosis de 0,25 L durante cinco minutos por plántula, cada siete días, obteniendo 10 dosis para todo el experimento. Se establecieron tres tiempos de exposición al flujo (7 1/2, 15 y 30 min), además se contó con un testigo recirculado sin el solenoide (T) y un testigo absoluto sin recircular que usó agua potable únicamente (Ta). Se empleó un diseño unifactorial completamente al azar. Los resultados indicaron que la exposición al campo durante 7 1/2 minutos generó diferencias estadísticamente significativas $(\mathrm{P} \leq 0,05)$ en las variables de respuesta en comparación con los tratamientos $\mathrm{T}$ y Ta, superándolos, respectivamente, en $18 \%$ y 40,1 \% para la longitud de la planta; en $80 \%$ y $98 \%$ para el número de rizomas; en 50 \% y $500 \%$ para el número de macollos; $73,7 \%$ y $217,4 \%$ para la masa fresca $\mathrm{y}$, finalmente, en un 70,1\% y $186,6 \%$ para la masa seca. Los resultados indican que el riego con bio-fertilizante estimulado magnéticamente puede considerarse como una tecnología complementaria para aumentar la producción de C. longa.

\section{Palabras clave}

Magnetobiología, tecnología agroambiental, campo magnético, C. longa, suelo.

\section{Abstract}

The growth and production response of turmeric (Curcuma longa L.) to the application of a mixture solution of bio-fertilizer Multibiol ${ }^{\circledR}$ and water, treated with a AC magnetic flux induction of $25 \mathrm{mT}$ and $60 \mathrm{~Hz}$ was evaluated. A circuit connected to a recirculation solenoid was used. The solution was applied in a concentration ratio of $1: 12$ to seedlings under greenhouse conditions for three months, at doses of $0.25 \mathrm{~L}$ for five min, every seven days, getting 10 doses for the entire experiment. Three periods of exposure to the flux $\left(7 \frac{1}{2}, 15\right.$ and $30 \mathrm{~min}$ ), a recirculated treatment without solenoid (T) and an absolute control without recirculate that used only potable water $(\mathrm{Ta})$ were established too. A univariate completely randomized design was used. The results indicated that the exposure to the field for $71 / 2$ min resulted in statistically significant differences $(\mathrm{P} \leq 0.05)$ in the response variables compared with the treatment $\mathrm{T}$ and $\mathrm{Ta}$, respectively, $18 \%$ and $40.1 \%$ for the length of the plant; in $80 \%$ and $98 \%$ for the number of rhizomes; in $50 \%$ and $500 \%$ for the number of shots; $217.4 \%$ and $73.7 \%$ for fresh mass and finally $70.1 \%$ and $186.6 \%$ for the dry mass. The results indicate that irrigation with magnetically stimulated bio-fertilizer can be considered as a complementary technology to increase the production of $C$. longa.

\section{Keywords}

Magnetobiology, agri-environment technology, magnetic field, C. longa, soil. 


\section{INTRODUCCIÓN}

La dependencia de fertilizantes de síntesis química para la producción agrícola competitiva es una de las mayores dificultades que enfrenta la agricultura convencional. Según el documento [1], se acepta que los fertilizantes son un componente importante en los costos de producción de la actividad agropecuaria, afectando directamente el precio de comercialización de los productos y el margen de ganancia del agricultor. El precio que debe pagar un agricultor por fertilizantes minerales en una zona remota es mucho más alto que el precio del mercado mundial a causa del transporte, distribución y otros costos de transacción asociados [2], lo que en muchas ocasiones no se traduce en un mayor valor de compra para el agricultor [3]. En este sentido se hace necesario impulsar el desarrollo de tecnologías innovadoras que propicien la disminución del uso de fertilizantes minerales a la vez que mejoren la eficiencia de los así llamados bio-fertilizantes.

La tecnología evaluada en la presente investigación corresponde al uso de un campo magnético (CMG) sobre una solución nutritiva preparada a partir del biofertilizante comercial Multibiol ${ }^{\circledR}$ (fabricado por la casa Agricultura Biológica en la ciudad de Buga, Valle del Cauca) y que fue disuelto en agua potable. Al parecer, la tecnología del magnetismo acelera la acción dinámica de los microorganismos benéficos sobre el suelo, realizando un proceso de rehabilitación químico-biológico sobre los suelos afectados por la salinidad, reduciendo el tiempo y aumentando la eficiencia del mejoramiento de las condiciones del suelo a través de la actividad biológica [4].

Los campos magnéticos (CMGs) afectan la dirección de la migración y alteran el crecimiento y la reproducción de los microorganismos, causan cambios en la síntesis de $\mathrm{ADN}$, en la orientación de bio-moléculas y bio-membranas y alteran el flujo de iones a través de la membrana plasmática, gene- rando como resultado una modificación en la velocidad de reproducción celular [5].

El efecto que generan los CMGs sobre el crecimiento celular se puede clasificar en inhibitorio, estimulatorio o no observable. Los efectos de estimulación o inhibición de los CMGs se han atribuido a cambios en la orientación de las bio-moléculas (proteínas), cambios en las bio-membranas (lipídicas y plasmáticas), alteraciones del flujo de iones a través de la membrana plasmática y/o cambios en la estructura de las biomoléculas [6].

Según [7] el mejor efecto sobre las partículas coloidales se obtiene cuando el medio líquido se hace pasar varias veces a través del dispositivo generador de campo, estos dispositivos utilizan densidades de flujo magnético relativamente altas $(0,01$ $0,5 \mathrm{~T})$ y son costosos. Una manera de disminuir costos es mediante el uso de bobinas o solenoides adaptados a un circuito hidráulico de recirculación, dentro del cual el fluido será expuesto a un CMG determinado [8]. A este respecto, [4] apuntaron que la estimulación magnética se debe realizar por solenoides. La generación de CMGs a través de bobinas mejora significativamente la estimulación, en relación a los CMGs (con imanes). El tratamiento de estimulación magnética complementa las llamadas técnicas duras (estimulación física con CMGs) con técnicas blandas (estimulación biológica con microorganismos)[4]. [9] determinó que la estimulación magnética de microorganismos benéficos aplicados a la cachaza acelera el proceso de mineralización del carbono y aumenta la respuesta agronómica de la planta indicadora.

Se han propuesto varias teorías para explicar el efecto de los CMGs débiles sobre los organismos y varios autores hacen mención a un fenómeno denominado "la resonancia del ciclotrón" como uno de los responsables de dichos efectos [5]. Los CMGs pueden activar las partículas coloidales del medio de cultivo, haciendo que estas remuevan parte del calcio unido a los 
fosfolípidos de las membranas plasmáticas, incrementando su permeabilidad y de esta forma, afectando el crecimiento de los microorganismos [8].

Hay diferencias significativas en los valores de inducción magnética de un CMG. Investigadores han trabajado con campos de $30 \mathrm{mT}$ [10] hasta $250 \mathrm{mT}$ [11] y a diferentes tiempos de exposición, desde 15 segundos [12] hasta 1 hora [13]. El efecto de diferentes dosis puede ser explicado también con las propiedades de los iones. Los iones en la célula tienen la habilidad de absorber energía magnética correspondiente a parámetros específicos relacionados a su vibración y a la rotación de energía en sus subniveles. Este fenómeno representa un tipo de absorción de resonancia y podría explicar el fuerte efecto que tiene la aplicación de valores definidos de CMGs [14].

La heterogeneidad de las especies y tipos de plantas, las condiciones físicoquímicas de los bio-fertilizantes y la calidad del agua de riego con la que se mezclará, los tipos de suelo, las condiciones locales y la falta de un modelo físico así como de unos protocolos estandarizados para el tratamiento de la tecnología de tratamiento magnético con fines agrícolas es muy compleja, de ahí que sean necesarias más investigaciones a nivel local para obtener información suficiente como para generar curvas detalladas que integren la inducción del CMG, el tiempo de exposición y la respuesta agronómica en cultivos promisorios, como lo es el de la $C$. longa, sobre planes de fertilización definidos y documentados.

El objetivo de esta investigación fue el de evaluar la respuesta de la $C$. longa a la aplicación de una mezcla en solución del bio-fertilizante Multibiol ${ }^{\circledR}$ y agua potable tratado con un CMG generado con $\mathrm{AC}$ y con una frecuencia de $60 \mathrm{~Hz}$.

\section{MATERIALES Y MÉTODOS}

La investigación se realizó bajo condiciones de invernadero en el vivero ILAMA ubicado en la granja experimental de la Escuela de Ingeniería de Recursos Naturales y del Ambiente EIDENAR de la Universidad del Valle, en la ciudad de Cali, la cual se encuentra a una altitud de 995 msnm y con coordenadas $3^{\circ} 22^{\prime} 33,55^{\prime \prime} \mathrm{N}$ y $76^{\circ} 31^{\prime} 58,43$ " O; cuenta con una temperatura promedio anual de $23^{\circ} \mathrm{C}$, humedad relativa del $73 \%$, precipitación promedio anual de $908 \mathrm{~mm}$ y 162 horas en promedio de brillo solar.

\subsection{Circuito hidráulico de recirculación}

El circuito hidráulico de recirculación para bio-fertilizante cuenta con un solenoide capaz de generar una inducción magnética de $100 \mathrm{mT}$ usando corriente alterna a una frecuencia de $60 \mathrm{~Hz}$. La intensidad del $\mathrm{CE}$ se reguló con un variador voltaje MCP Lab. Electronics M10-522-30 y un multímetro UNI-T UT61C RS232C. El solenoide fue fabricado en el Laboratorio de Mecánica Fina adscrito a la Facultad de Ciencias Naturales y Exactas de la Universidad del Valle campus Meléndez, el diámetro interno de la bobina es de $2,54 \mathrm{~cm}$, su longitud es de $5,00 \mathrm{~cm}$, la bobina cuenta con 30 capas y 29 espiras de alambre de cobre calibre 14. El marco fue fabricado de PVC con cara cuadrada de 19,30 $\mathrm{cm}$ de longitud y $1,50 \mathrm{~cm}$ de grosor con un orificio central de $2,54 \mathrm{~cm}$ de diámetro para permitir la conexión con el circuito hidráulico de recirculación de agua (Fig. 1). 


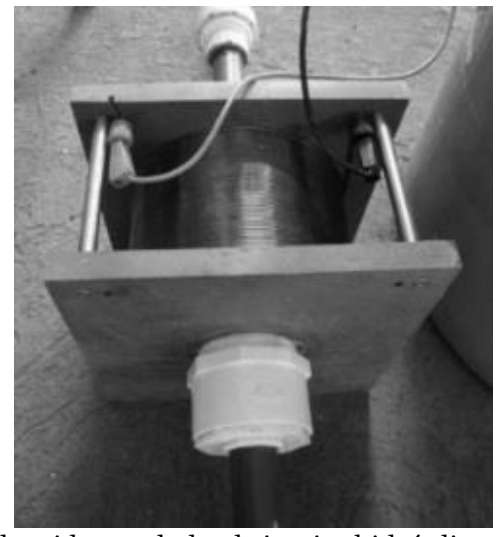

Fig. 1. Solenoide acoplado al circuito hidráulico de recirculación de bio-fertilizante tratado magnéticamente. Fuente: Autores.

El circuito hidráulico de recirculación contó con una electrobomba Titán QB60 con caudal máximo de $30 \mathrm{~L} \mathrm{~min}^{-1}$. Para el almacenamiento del agua se utilizó un recipiente plástico de $0,10 \mathrm{~m}^{3}$ y un sistema de tubería de PVC de $2,54 \mathrm{~cm}$ de diámetro con válvulas de control de flujo (Fig. 2).

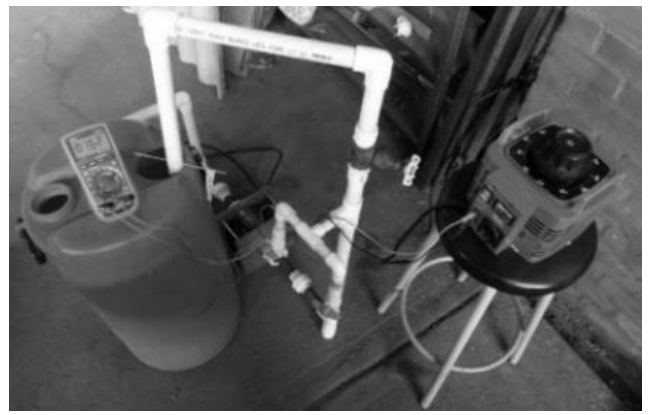

Fig 2. Circuito hidráulico de recirculación de biofertilizante tratado magnéticamente. Fuente: Autores.

\subsection{Diseño experimental}

El ensayo se ajustó a un diseño unifactorial de múltiples niveles dispuestos completamente al azar con el factor: tiempo de recirculación con un $\mathrm{CMG}$ de $(25 \mathrm{mT})$ y tres niveles (7 1/2, 15 y $30 \mathrm{~min})$. Se contó con un testigo (T) que fue recirculado sin el solenoide acoplado al sistema (i.e. sin estimular) y un testigo absoluto (Ta) que no fue recirculado y se obtuvo de un sistema de riego alterno que usó agua potable (Tabla 1).
Tabla 1. Tratamientos implementados para bio-fertilizante tratado magnéticamente. Fuente: Autores.

\begin{tabular}{cc}
\hline Número & Tratamiento \\
\hline 1 & $25 \mathrm{mT} 7 \frac{1}{2} \mathrm{~min}$ \\
2 & $25 \mathrm{mT} 15 \mathrm{~min}$ \\
3 & $25 \mathrm{mT} 30 \mathrm{~min}$ \\
4 & $\mathrm{~T}$ \\
5 & $\mathrm{Ta}$ \\
\hline
\end{tabular}

Para el desarrollo del montaje experimental se preseleccionaron rizomas de $C$. longa, provenientes de la vereda "El Limonar" en el municipio de Dagua, Valle del Cauca. El criterio de selección de los rizomas correspondió a dos características fundamentales: condiciones fitosanitarias y dimensiones similares (longitud, diámetro) para tener unidades experimentales sanas y homogéneas. Los rizomas de cúrcuma se sembraron en arena de río corriente que se ubicó en camas de pre-germinación de plástico. Los rizomas se diferenciaron con códigos correspondientes a su tratamiento y a su repetición, ubicándose en distintas camas de pre-germinación. Los rizomas se pre-germinaron durante un mes. Se regó a las unidades experimentales con el biofertilizante estimulado magnéticamente de acuerdo con su código, las unidades experimentales correspondientes a los tratamientos testigo y testigo absoluto se regaron con bio-fertilizante sin estimular y con agua potable, respectivamente, en su cama de pre-germinación correspondiente. Una vez que los rizomas produjeron un brote de dos cm de longitud y una hoja verdadera, se procedió a su trasplante a una bolsa negra plástica de vivero calibre cuatro que previamente se llenó con $15 \mathrm{~kg}$ de suelo proveniente de la vereda El Limonar; en la Tabla 2 se exponen los resultados de la caracterización físico-química del suelo utilizado. 
Respuesta de la Cúrcuma (Curcuma longa L.) a la aplicación de un bio-fertilizante tratado con un campo magnético

Tabla 2. Caracterización físico-química del suelo utilizado. Fuente: Autores

\begin{tabular}{|c|c|c|c|c|c|c|c|c|c|c|c|c|c|c|c|}
\hline Arena & Limo & Arcilla & $\mathrm{CO}$ & \multirow{2}{*}{ Textura } & \multirow{2}{*}{$\mathrm{pH}$} & $\mathrm{CE}$ & $\mathrm{P}$ & $\mathrm{S}$ & $\mathrm{Fe}$ & $\mathrm{K}^{+}$ & $\mathrm{Ca}$ & $\mathrm{Mg}$ & $\mathrm{Na}$ & $\mathrm{Al}$ & CICE \\
\hline \multicolumn{4}{|c|}{$\%$} & & & $\mathrm{dS} \mathrm{m}^{-1}$ & \multicolumn{3}{|c|}{$\mathrm{mg} \mathrm{kg-1}$} & \multicolumn{6}{|c|}{$\mathrm{cmol}^{(+)} \mathrm{kg}^{-1}$} \\
\hline 40 & 22 & 38 & 2,4 & Fr-Ar & 5,2 & 0,12 & 1,3 & 7,2 & 150 & 0,2 & 3,8 & 3,6 & 0,2 & 0,07 & 7,9 \\
\hline
\end{tabular}

Métodos: textura: Bouyoucos; pH: pasta saturación; CE: extracto saturación; CICE: suma de cationes; CO: Walkley-Black; P: Bray II; S: fosfato monocálcico; K, Ca, Mg, Na: $\mathrm{AcONH}_{4}$; Fe: Mehlich

Se dispusieron tres réplicas para el $\mathrm{T}$ y el Ta y cinco réplicas para los tres tratamientos restantes, obteniendo un total de 21 unidades experimentales. Para la aplicación de los tratamientos se conectó el circuito de recirculación a un sistema de riego localizado de alta frecuencia, lo que permitió la aplicación inmediata de los tratamientos a las plántulas. Se utilizó una manguera de 1,60 cm para conducir el biofertilizante tratado magnéticamente desde el circuito hasta las plantas, se utilizaron goteros con caudal medio de $2 \mathrm{~L} \mathrm{~h}^{-1}$. Se regó con una frecuencia fija de aplicación de 7 días. La mezcla de agua más biofertilizante se determinó con base a las recomendaciones de uso por el fabricante, usándose una concentración agua y biofertilizante de $12: 1$, es decir, se utilizaron $12 \mathrm{~L}$ de agua potable para disolver $1 \mathrm{~L}$ de Multibiol®. La cantidad de solución aplicada a cada plántula fue de $0,25 \mathrm{~L}$ durante 5 minutos lapso bajo el cual el suelo llegó a capacidad de campo.

Se evaluaron las siguientes variables de respuesta: longitud de la planta en $\mathrm{cm}$, número de rizomas, número de macollos, masa fresca y masa seca en $\mathrm{kg}$. La medición de la longitud de las plantas se realizó a los 5 días de trasplantadas y luego se realizó cada 3 a 10 días, tomando en consideración la distancia entre el nivel del suelo de la matera hasta el punto de inserción de la hoja central más joven de cada planta. La cosecha se llevó a cabo 109 días después de la siembra. La masa fresca se midió con la balanza analítica OHAUS Traveler, posteriormente, las plantas se trocearon y se empacaron por separado en bolsas de papel. Las bolsas se deshidrataron durante 7 días a una temperatura de $90{ }^{\circ} \mathrm{C}$ en el horno Quincy Lab. Inc. 40GC, garantizando así un porcentaje de humedad cercano al $10 \%$. Se registró la masa seca con la balanza analítica OHAUS Traveler.

\subsection{Actividad microbiana en muestras de suelo}

Durante la cosecha, se tomaron tres muestras de suelo a profundidades de 10,00 a $15,00 \mathrm{~cm}$ de cada uno de los cinco tratamientos sobre las cuales se desarrolló la cúrcuma, incluyendo a los tratamientos testigo; a las tres repeticiones por tratamiento se les realizó la prueba de actividad microbiana con un tiempo de incubación de 6 días.

Para determinar la actividad microbiana del suelo luego de la cosecha, se siguió el método CAB del Centro de Agrobiología del Brasil, indicado por [15]: se incubaron $50 \mathrm{~g}$ de suelo durante 8 días a $24^{\circ} \mathrm{C}$ en un recipiente de cierre hermético en donde también se ubicaron $10 \mathrm{ml}$ de hidróxido de sodio $(\mathrm{NaOH}) 1 \mathrm{~N}$ el cual atrapa el dióxido de carbono $\left(\mathrm{CO}_{2}\right)$ desprendido por la muestra, formando carbonato de sodio. Además, se preparó una muestra testigo incubada sin presencia de suelo. Después de 5 días de incubación se adicionaron $2 \mathrm{ml}$ de cloruro de bario $\left(\mathrm{BaCl}_{2}\right)$ al $10 \%$ para precipitar el $\mathrm{CO}_{2}$ en forma de carbonato de bario $\left(\mathrm{BaCO}_{3}\right)$. Luego se adicionaron dos gotas de fenolftaleína $1 \%$ (solución alcohólica indicadora) y se tituló con ácido clorhídrico ( $\mathrm{HCl})$ 0,5 N lentamente para cuantificar el volumen de $\mathrm{NaOH}$ que no reaccionó con el $\mathrm{CO}_{2}$. El blanqueamiento de la solución titulada indicó la neutralización de todo el hidróxido. Análogamente la muestra testigo se tituló con $\mathrm{HCl}$ 0,5 N. 
La determinación de la actividad microbiana para las muestras de suelo, se realizó calculando el contenido de $\mathrm{CO}_{2}$ de acuerdo a (1):

$A M S=\left[\frac{(B-T) * N H C l * 0,006}{P}\right] * 10^{6}=\left[\frac{\mu g C-C O_{2}}{g S S}\right]$

Donde, la AMS es la actividad microbiana del suelo, B es la titulación en blanco, $\mathrm{T}$ es la titulación muestra de suelo, $\mathrm{N}$ $\mathrm{HCl}$ es la normalidad del ácido, $\mathrm{P}$ es la masa seca de la muestra y 0,006 es el factor de dilución.

\subsection{Análisis estadístico}

Se realizó un análisis de varianza a los datos obtenidos con un nivel de confianza del $95 \%$ y una vez verificada, se siguió con la comparación entre medias con el test de Tukey $(\mathrm{P} \leq 0,05)$ con el paquete estadístico Minitab® versión 16 .

\section{RESULTADOS Y DISCUSIÓN}

Los resultados indicaron un efecto benéfico sobre las variables de respuesta analizadas (altura de la planta, número de rizomas, número de macollos, masa fresca y masa seca), el cual se observó en el menor tiempo de exposición al CMG.

\subsection{Longitud de la planta}

El tratamiento $25 \mathrm{mT}-7 \frac{1}{2} \mathrm{~min}$ presentó los mejores resultados en todas las mediciones realizadas en el periodo de evaluación. Este tratamiento generó diferencias estadísticamente significativas $(\mathrm{P} \leq 0,05)$ en las variables de respuesta en comparación con los tratamientos $\mathrm{T}$ y Ta, superando al $\mathrm{T}$ y al Ta, respectivamente, en $18 \% \mathrm{y}$ $40,1 \%$ para la longitud de la planta (Fig. $3)$.

En [16] encontraron que el uso de agua de riego tratada magnéticamente (ATM) incrementó significativamente las concen- traciones de $\mathrm{K}^{+}$sobre el suelo en comparación al testigo, donde la mayor concentración de $\mathrm{K}^{+}$se halló a una profundidad de 15,00 a $30,00 \mathrm{~cm}$ mientras que las menores concentraciones se encontraron en la superficie del suelo $(0,00-5,00 \mathrm{~cm})$. La toma de nutrientes por la cúrcuma tiene el siguiente orden: $\mathrm{K}^{+}>\mathrm{N}>\mathrm{Mg}>\mathrm{Ca}$ y la producción promedio de rizomas secos de 5,5 toneladas por hectárea remueve $91,00 \mathrm{~kg}$ de $\mathrm{N}, 16,90 \mathrm{~kg}$ de $\mathrm{P}_{2} \mathrm{O}_{5}$ y $245,00 \mathrm{~kg}$ de $\mathrm{K}^{+}$ [17], lo que demuestra que este cultivo consume altas cantidades de $\mathrm{K}^{+}$para su desarrollo fisiológico. El suelo contó con niveles medios de $\mathrm{K}^{+}$[18], [19] (Tabla 2), no obstante, fue con el tiempo de exposición de 7 1 $1 / 2$ minutos con el que la planta alcanzó una mayor altura con respecto a los demás tratamientos (Fig. 3), esto podría indicar un aumento en la disponibilidad del $\mathrm{K}^{+}$ debido al riego con bio-fertilizante estimulado magnéticamente, hecho que concuerda con resultados obtenidos por [20].

Los CMGs han sido reportados por su influencia tanto en la activación de iones como con la polarización de los dipolos en la célula viva [11]. Los CMGs pueden alterar la estructura y función de la membrana plasmática de los microorganismos de manera negativa, lo cual podría explicar el porqué de los menores resultados en todas las variables de respuesta obtenidas al exponer los microorganismos durante un mayor tiempo al CMG. Existen investigaciones que indican que a bajas densidades de flujo magnético y menores tiempos de exposición se pueden lograr resultados positivos, [4] estimularon una mezcla de bio-fertilizantes líquidos (Multibiol, Biosol New, Desalt, Biocompost y micorrizas) sometida a un CMG de $4 \mathrm{mT}$ durante dos horas para ser aplicado a un cultivo de maíz (Zea mays),apreciando mayor número y tamaño de mazorcas con este tratamiento que además aumentó significativamente la biomasa producida por el grano de maíz, los autores concluyeron que la aplicación de bio-fertilizante tratado magnéticamente no sólo tiene los beneficios de los procesos 
biológicos de los microorganismos en el enriquecimiento del suelo, sino que también acelera este proceso con la aplicación de CMGs para aumentar la eficacia y reducir el tiempo de mejoramiento del suelo, esto se debe a que al aplicar CMGs alternos al sistema de fertirriego se disminuye la tensión superficial de la solución salina en el suelo, aumentando la solubilidad de las sales y mejorando los procesos dinámicos de infiltración y movilidad de iones, lo cual probablemente explique el mejor comportamiento del bio-fertilizante tratado magnéticamente con respecto a los otros tratamientos. Por otro lado, el incremento de la altura en el tratamiento $25 \mathrm{mT}$ - $7 \frac{1}{2}$ min, con respecto a los demás tratamientos, puede estar asociado al nivel de materia orgánica que contiene el suelo que después de las aplicaciones de bio-fertilizante tratado magnéticamente; al menor tiempo pudo haber dado pie al desarrollo de un efecto sinérgico entre los microorganismos ya presentes en el suelo, los adicionados en la solución estimulada y los contenidos de macro y microelementos presentes en el suelo para darle ventaja sobre los otros tratamientos. $\mathrm{Al}$ respecto, es importante mencionar que la presencia de material orgánico, determinado con el método de Walkley-Black para suelos, es el factor que más influye en la actividad y población de microorganismos del suelo, ya que la materia orgánica es una fuente de energía y los suelos de El Limonar cuentan con niveles normales-altos (Tabla 2).

\subsection{Número de rizomas}

Se verificó la dispersión de los datos con estadísticos descriptivos (Tabla 3) para las variables de respuesta: número de rizomas, número de macollos, masa fresca y masa seca en $\mathrm{kg}$.

Se utilizó la prueba de Tukey $(\mathrm{P} \leq 0,05)$ para determinar el tratamiento que produjo mayor diferencia estadísticamente significativa para cada una de las variables de respuesta (Tabla 4).

El tratamiento que mostró el mayor número de rizomas y con mayor significancia estadística según la prueba de Tukey (P $\leq 0,05)$ corresponde al tratamiento $25 \mathrm{mT}$ $71 / 2 \mathrm{~min}$, que superó al Ta en términos de número promedio de rizomas en un $98 \% \mathrm{y}$ a la vez superó al T en el número promedio de rizomas en un $80 \%$ (Fig. 4).

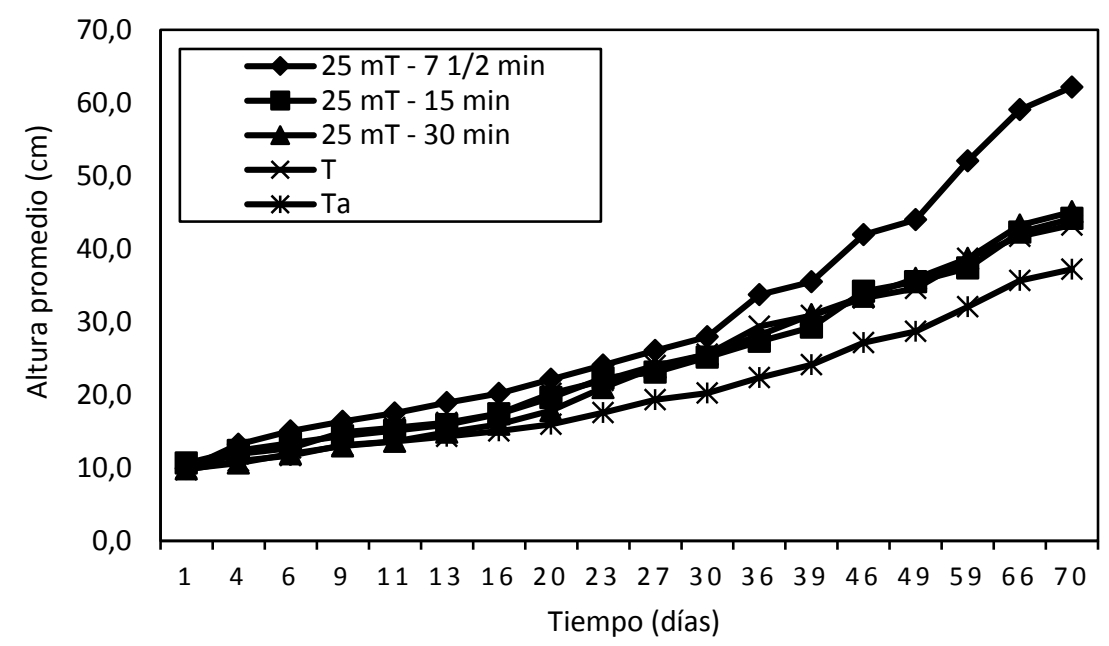

Fig 3. Respuesta de la altura promedio de la planta $(\mathrm{cm})$ versus tiempo (días) para los distintos tratamientos. Fuente: Autores. 
Respuesta de la Cúrcuma (Curcuma longa L.) a la aplicación de un bio-fertilizante tratado con un campo magnético

\begin{tabular}{lcccc}
\multicolumn{4}{c}{ Tabla 3. Estadísticos descriptivos para las variables de respuesta. Fuente: Autores. } \\
\hline Estadístico & $\begin{array}{c}\text { Número de } \\
\text { rizomas }\end{array}$ & $\begin{array}{c}\text { Número de } \\
\text { macollos }\end{array}$ & $\begin{array}{c}\text { Masa fresca } \\
(\mathrm{kg})\end{array}$ & $\begin{array}{c}\text { Masa seca } \\
(\mathrm{kg})\end{array}$ \\
\hline Media & 8,6 & 4,5 & 0,502 & 0,054 \\
Error estándar de la media & 0,7 & 0,4 & 0,036 & 0,004 \\
Desviación estándar & 3,0 & 1,8 & 0,163 & 0,016 \\
Varianza & 9,2 & 3,1 & 0,027 & 0,271 \\
Coeficiente de variación & 35,3 & 39,1 & 0,033 & 0,031 \\
Mínimo & 5,0 & 0,0 & 0,184 & 0,022 \\
Mediana & 8,0 & 5,0 & 0,491 & 0,059 \\
Máximo & 16,0 & 7,0 & 0,787 & 0,079 \\
\hline
\end{tabular}

Tabla 4. Efecto de los tratamientos sobre las variables de respuesta bajo condiciones de invernadero. Promedios con letras distintas, en la misma columna y clasificados por tratamientos, indican

\begin{tabular}{lcccc}
\multicolumn{4}{c}{ diferencia significativa según la prueba de Tukey $(\mathrm{P} \leq 0,05)$. Fuente: Autores. } \\
\hline Tratamiento & $\begin{array}{c}\text { Número de } \\
\text { rizomas }\end{array}$ & $\begin{array}{c}\text { Número de } \\
\text { macollos }\end{array}$ & $\begin{array}{c}\text { Masa fresca } \\
(\mathrm{kg})\end{array}$ & $\begin{array}{c}\text { Masa seca } \\
(\mathrm{kg})\end{array}$ \\
\hline $25 \mathrm{mT}-30 \mathrm{~min}$ & $13,2 \mathrm{a}$ & $6 \mathrm{a}$ & $0,699 \mathrm{a}$ & $0,072 \mathrm{a}$ \\
$25 \mathrm{mT}-15 \mathrm{~min}$ & $8,0 \mathrm{~b}$ & $5 \mathrm{ab}$ & $0,540 \mathrm{~b}$ & $0,057 \mathrm{~b}$ \\
$25 \mathrm{mT}-71 / 2 \mathrm{~min}$ & $7,3 \mathrm{~b}$ & $4,8 \mathrm{ab}$ & $0,494 \mathrm{~b}$ & $0,054 \mathrm{~b}$ \\
$\mathrm{Ta}$ & $6,7 \mathrm{~b}$ & $4 \mathrm{~b}$ & $0,402 \mathrm{~b}$ & $0,042 \mathrm{bc}$ \\
$\mathrm{T}$ & $6,4 \mathrm{~b}$ & $1 \mathrm{c}$ & $0,220 \mathrm{c}$ & $0,025 \mathrm{c}$
\end{tabular}

Con respecto a número de macollos, el tratamiento $25 \mathrm{mT}-7 \frac{1}{2} \mathrm{~min}$ superó tanto al Ta como al T en un $500 \%$ y $50 \%$ respectivamente (Fig. 4). [21] al evaluar el efecto del ATM a una intensidad de $30 \mathrm{mT}$ aplicada sobre un cultivo de fríjol común (Phaseolus vulgaris L.), concluyó que esta puede mejorar la cantidad y calidad del cultivo, por otro lado, el estudio sugiere que el ATM podría estimular el sistema de defensa, actividad fotosintética y la eficiencia de translocación de los fotoasimilados en esta planta, lo que sumado a la carga de microorganismos adicionados al suelo, podría redundar en el desarrollo de una planta de mayor tamaño y con producciones de rizomas superiores a los demás tratamientos analizados.

\subsection{Masa fresca y masa seca}

La prueba de Tukey $(\mathrm{P} \leq 0,05)$ indicó que la mejor masa fresca correspondió al tratamiento $25 \mathrm{mT}$ - 7 1⁄2 min el cual superó en promedio al Ta y al T en $217,4 \%$ y 73,7 $\%$, respectivamente.

La prueba de Tukey $(\mathrm{P} \leq 0,05)$ indicó que la mejor masa seca correspondió al tratamiento $25 \mathrm{mT}$ - $7 \frac{1}{2} \mathrm{~min}$ el cual superó en promedio al Ta y al $\mathrm{T}$ en $186,6 \% 70,1 \%$ respectivamente.

\subsection{Actividad microbiana}

Los datos encontrados superaron los resultados de varias investigaciones que establecieron rangos de respiración obtenidos en laboratorio, para suelos agrícolas (15-32 $\mu \mathrm{gC}-\mathrm{CO}_{2}$ gSS $\left.^{-1}\right)$ y suelos enmendados (53-86 $\left.\mu \mathrm{gC}-\mathrm{CO}_{2} \mathrm{gSS}^{-1}\right)$ [22] donde los mayores valores llegaron hasta $85,1 \mu \mathrm{gC}$ $\mathrm{CO}_{2}$ gSS $^{-1}$ mientras que para este trabajo de investigación el mayor valor registrado fue $184 \mu \mathrm{gC}-\mathrm{CO}_{2} \mathrm{gSS}^{-1}$, cabe resaltar que este valor está asociado al tratamiento 25 $\mathrm{mT}$ - 7 1/2 min que mostró los mejores resultados de las variables de respuesta analizadas (Tabla 5). 


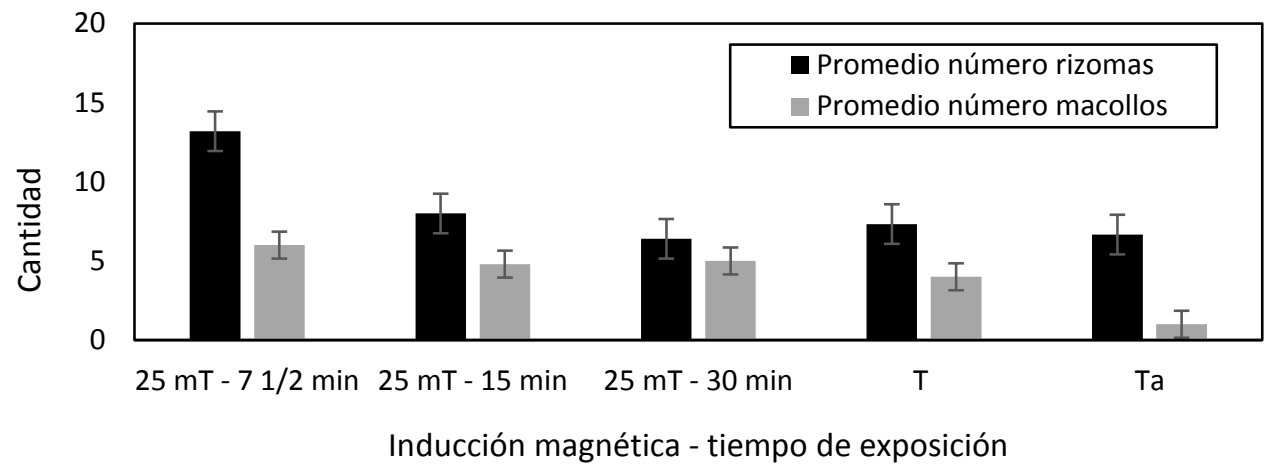

Fig. 4. Número de rizomas y macollos promedio por tratamiento. Fuente: Autores.

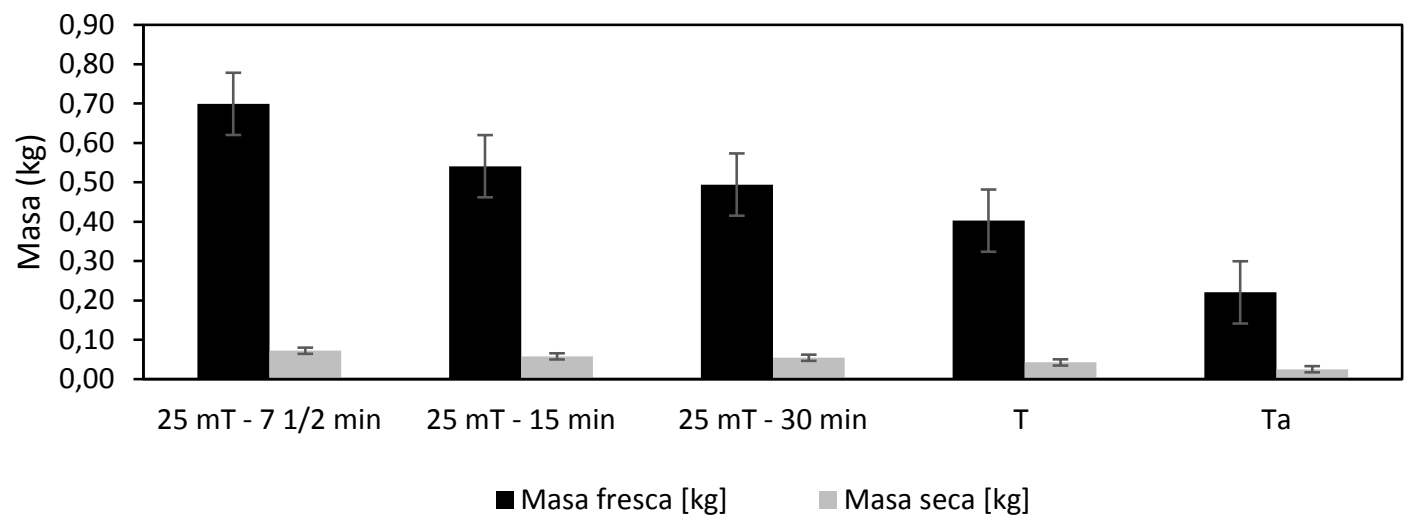

Fig. 5. Masa fresca y seca (kg) de la plántula de cúrcuma. Fuente: Autores.

La planta en sí misma juega un papel crítico como estimulador de los microorganismos en el suelo, los cuales captan las exudaciones radicales o desprendimiento de materiales orgánicos. Es interesante notar que el tratamiento $25 \mathrm{mT}-7 \frac{1}{2} \mathrm{~min}$ fue el que produjo la mayor actividad microbiana (Tabla 5) y que, a su vez, este tratamiento tuvo asociado la mayor cantidad de rizomas y de macollos en promedio, debido probablemente a que en la rizósfera de la cúrcuma hay mayor concentración de nutrientes orgánicos provenientes de las raíces, las cuales favorecen el crecimiento de los microorganismos que a su vez mejoran la asimilación de nutrientes para las plantas.

El mejor resultado obtenido sobre las variables de respuesta analizadas (altura de la planta, número de rizomas, número de macollos, masa fresca y masa seca) fue obtenido en el tratamiento $25 \mathrm{mT}$ - 7 1/2 min con un CMG de $25 \mathrm{mT}$ producido con $\mathrm{AC}$ con una frecuencia de $60 \mathrm{~Hz}$.

\section{CONCLUSIONES}

Los resultados de esta investigación indican que el riego con bio-fertilizante estimulado magnéticamente puede ser considerado como una tecnología complementaria utilizada para aumentar potencialmente el rendimiento del cultivo de la cúrcuma al incidir positivamente sobre las propiedades físicas, químicas y biológicas del suelo, no obstante lo anterior, se requieren estudios adicionales que permitan sesgar de manera más precisa el efecto de diversos campos magnéticos sobre la población microbiana de enmiendas o fertilizantes de origen biológico aplicados sobre el suelo. 
Respuesta de la Cúrcuma (Curcuma longa L.) a la aplicación de un bio-fertilizante tratado con un campo magnético

\begin{tabular}{lcc} 
Tabla 5 . Actividad microbiana en muestras de suelo por tratamiento. Fuente: Aut \\
\cline { 2 - 3 } Tratamiento & $\begin{array}{r}\text { Actividad Microbiana } \\
\text { Promedio [ } \mu \mathrm{gC}-\mathrm{CO} 2 / \mathrm{gSS}]\end{array}$ & $\begin{array}{c}\text { Desviación } \\
\text { estándar }\end{array}$ \\
\hline $25 \mathrm{mT}-71 / 2$ minutos & 184 & 6,928 \\
$25 \mathrm{mT}-15$ minutos & 138 & 18,33 \\
$25 \mathrm{mT}-30$ minutos & 122 & 36,497 \\
$\mathrm{~T}$ & 126 & 6,928 \\
$\mathrm{Ta}$ & 124 & 15,100 \\
\hline
\end{tabular}

\section{AGRADECIMIENTOS}

La presente investigación se realizó con recursos provenientes del Sistema General de Regalías en el marco del proyecto "Desarrollo de un Sistema Agroindustrial Rural Competitivo en una Bio-región del Valle del Cauca" a cargo del Grupo de Investigación en Ciencias Ambientales y de la Tierra ILAMA, adscrito al Departamento de Física perteneciente a la Facultad de Ciencias Naturales y Exactas de la Universidad del Valle, sede Meléndez, Cali Colombia.

\section{REFERENCIAS}

[1] CONPES. 2009. Documento CONPES 3577. Política nacional para la racionalización del componente de costos de producción asociado a los fertilizantes en el sector agropecuario. En: Asociación Nacional de Empresarios de Colombia (ANDI)

[2] J. Kotschi, A soiled reputation. Adverse impacts of mineral fertilizers in tropical agriculture, $1^{\text {st }}$ ed. Berlin, Germany: Heinrich Böll Stiftung (Heinrich Böll Foundation) Publisher, 2015.

[3] J.P. Pérez, "Uso de los fertilizantes y su impacto en la producción agrícola," Tesis de maestría. Depto. Biocien., Univ. Nac. Col., Medellín, Colombia, 2014.

[4] O. Zúñiga, J. Osorio, R. Cuero and J. Peña, "Evaluación de tecnologías para la recuperación de suelos degradados por salinidad," Rev. Fac. Nac. Agron., vol. 64, no. 1, pp. 57695779, Apr. 2011.

[5] J. Zapata, G. Moreno and E. Márquez, "Efectos de los campos magnéticos sobre el crecimiento de Saccharomyces cerevisiae," Interciencia, vol. 27, no. 10, pp. 544-550, Aug. 2002.

[6] U. Pothakamury, G. Barbosa and B. Swanson, "Magnetic Fields Inactivation of Microogan- isms and Generation of Biological Changes," Food Technol., vol. 47, no. 12, pp. 85-93, 1993.

[7] J. Baker and S. Judd, "Magnetic amelioration of scale formation," Water Res., vol. 30, no. 2, pp. 247-260, 1996.

[8] A. Goldsworthy, H. Whitney and E. Morris, "Biological effects of physically conditioned water," Water Res., vol. 33 no. 7, pp. 16181626, 1999.

[9] O. Zúñiga, R. Cuero and J. Peña, "Estimulación con campo electromagnético variable de microorganismos benéficos aplicados a la cachaza para mejorar su uso como biofertilizante," Rev. Bio. Agro., vol. 9, no. 2, pp. 150158, Aug. 2011.

[10] J.O. Odhiambo, F.G. Ndiritu and I.N. Wagara, "Effects of static electromagnetic fields at 24 hours incubation on the germination of Rose Coco Beans (Phaseolus vulgaris)," Romanian J. Biophys., vol. 19, no. 2, pp. 135-147. Apr. 2009.

[11] I.M. Ahmed, "Effects of magnetized low quality water on some soil properties and plant growth," Int. J. Res. Chem. Env., vol. 3, no. 2, pp. 140-147, 2013.

[12] S. Muszyński, M. Gagoś and S. Pietruszewski, "Short-Term Pre-Germination Exposure to ELF Magnetic Field Does Not Influence Seedling Growth in Durum Wheat (Triticum durum)," Polish J. of Environ. Stud., vol. 18, no. 6, pp. 1065-1072, 2009.

[13] E. Hincapié, J. Torres and L. Bueno, "Estudio de la estimulación magnética de semillas de Leucaena leucocephala (Lam.) de Wit," Tecno Lógicas, no. 29, pp. 33-47, 2012.

[14] A. Aladjadjiyan, "Physical Factors for Plant Growth Stimulation Improve Food Quality", in Food Production - Approaches, Challenges and Tasks, $1^{\text {st }}$ ed. Povdiv, Bulgaria: Intech, 2012.

[15] H. Montenegro and D. Malagón, Propiedades físicas de los suelos, 1st ed. Bogotá, Colombia: Sub-dirección agrológica Instituto Geográfico Agustin Codazzi. 1990.

[16] M.H. Hilal, Y.M. El-Fakhrani, S.S. Mabrouk, A.I. Mohamed and B.M. Ebead, "Effect of magnetic treated irrigation water on salt 
removal from a sandy soil and on the availability of certain nutrients," Int. J. Eng. App. Sci., vol. 2 no. 2, pp. 36-44, 2013.

[17] A.K. Sadanandan and S. Hamza, "Response of four turmeric (Curcuma longa L.) varieties to nutrients in an oxisol on yield and curcumin content," J. Plantation Crops, vol. 24, pp. 120-125, 1996

[18] A. Rojas, M. Ramírez, R. Lora, E. Amézquita and L. Sánchez, Fertilización en diversos cultivos: quinta aproximación, $1^{\text {st }}$ ed. Tibaitatá, Colombia: Produmedios, 1992.

[19] M. Andrades and M.E. Martínez. Fertilidad del suelo y parámetros que la definen, $3^{\text {rd }}$ ed., La
Rioja, España: Universidad de la Rioja, Servicio de Publicaciones, 2014.

[20] S. Mahmood and M. Usman, "Consequences of magnetized water application on Maize seed emergence in sand culture," J. Agr. Sci. Tech. vol. 16, pp. 47-55, 2014.

[21] H.R. Moussa, "The Impact of Magnetic Water Application for Improving Common Bean (Phaseolus vulgaris L.) Production," New York Sci. J., vol. 4 no. 6, pp. 15-20, 2011.

[22] J.A. Pascual, C. García and T. Hernandez, "Comparison of fresh and composted organic waste in their efficacy for the improvement of arid soil quality," Biores. Techn., vol. 68, no. 3, pp. 255-264, 1999. 